\section{Elastographie, transiente}

\author{
A. M. Gressner ${ }^{1}$ und O. A. Gressner ${ }^{2}$ \\ ${ }^{1}$ Labor Dr. Wisplinghoff Berlin, Berlin, Deutschland \\ ${ }^{2}$ Labor Dr. Wisplinghoff Köln, Köln, Deutschland
}

Synonym(e) Gewebeelastizitätsbestimmung der Leber

Englischer Begriff transient elastography, fibroscan

Definition Zur nicht invasiven Diagnostik der Leberfibrose/zirrhose eingesetzte Technik, die die durch den im Leberparenchym erhöhten Bindegewebsanteil veränderte Ausbreitungsgeschwindigkeit ultraschallinduzierter elastischer Wellen misst.

Beschreibung Die von einer auf der Leberoberfläche handgeführten, beweglichen Sonde ausgesendeten Ultraschallwellen niedriger Frequenz (50 MHz) und Amplitude werden in das Gewebe transmittiert. Die von den Vibrationswellen induzierten elastischen Scherwellen durchsetzen das Gewebe mit einer Geschwindigkeit, die direkt mit der Lebersteifigkeit korreliert: Je steifer/härter das Gewebe, desto schneller brei- ten sich die Wellen aus $(\mathrm{kPa})$. Von der Sonde wird in der Regel ein Gewebszylinder von ca. $1 \mathrm{~cm}$ Durchmesser und $5 \mathrm{~cm}$ Länge gemessen, was etwa dem 100-fachen Volumen einer perkutanen Leberbiopsie entspricht.

Diese nicht invasive Methode erlaubt die zuverlässige und reproduzierbare Diagnose einer signifikanten Fibrose und der Zirrhose, ist schnell, unbelastend, kostensparend und erfasst einen relativ großen Anteil des Organs. Limitation sind: starke Adipositas und Aszites, akute Hepatitis.

Je nach Grenzwert $(\mathrm{kPa})$ betragen die diagnostische Sensitivitäten ( $\triangleright$ Sensitivität, diagnostische) 63-90\%, Spezifitäten ( $\triangleright$ Spezifität, diagnostische) $82-97 \%$ und positive/negative Vorhersagewerte ( $\vee$ Vorhersagewert, positiver; $\triangleright$ Vorhersagewert, negativer) 71-91 \% bzw. 84-95\% (• Fibrosekenngrößen).

\section{Literatur}

Erhard A, Lörke J, Vogt C et al (2006) Transiente Elastografie zur Diagnostik der Leberzirrhose. Dtsch Med Wochenschr 131:2765-2769 Manning DS, Afdhal NH (2008) Diagnosis and qantitation of fibrosis. Gastroenterology 134:1670-1681 